\title{
EFFECTS OF CHARACTERISTIC MATERIAL LENGTHS ON DUCTILE CRACK PROPAGATION
}

\author{
E. Radi \\ Dipartimento di Scienze e Metodi dell'Ingegneria \\ Università di Modena e Reggio Emilia \\ Viale Allegri, 13. I-42100 Reggio Emilia, Italia. E-mail: eradi@unimore.it
}

\begin{abstract}
The asymptotic fields near the tip of a crack steadily propagating in a ductile material under Mode III loading conditions are investigated by adopting an incremental version of the indeterminate theory of couple stress plasticity displaying linear strain hardening. The adopted constitutive model is able to account for the microstructure of the material by incorporating two distinct material characteristic lengths. It can also capture the strong size effects arising at small scales, which results from the underlying microstructures. The effects of microstructure on Mode III crack tip fields mainly consist in a substantial increase in the singularities of the skew-symmetric stress and couple stress fields, which occurs also for small values of the strain hardening coefficient, whereas the symmetric stress field turns out to be non-singular according to the asymptotic crack tip fields for a stationary crack provided by the indeterminate theory of couple stress elasticity. The performed asymptotic analysis thus predicts a significant increase of the tractions level ahead of the crack-tip, due to the contribution of the rotation gradient.
\end{abstract}

\section{Introduction}

Due to the lack of a length scale, classical plasticity theories are not able to characterize the constitutive behavior of ductile materials at the micron scale. This lack is expected to be particularly significant for the analysis of the stress and deformation fields very near the tip of a crack propagating in a ductile metal, which are altered by the presence of the microstructure. In particular, experimental observations performed by Elssner et al. [1] found that during crack propagation the interface between a ductile crystal of niobium and a sapphire single crystal remained sharp and not blunted up to the atomic scale. They also found that the stress level required to produce atomic decohesion of the lattice turns out to be about 10 times the tensile yield stress, whereas fracture mechanics analyses based on classical plasticity theories provide a maximum stress level near a crack tip not larger than 4-5 times the tensile yield stress [2]. Therefore, for the investigations of the crack tip fields at the micron scale it becomes necessary to adopt enhanced constitutive models, which account for the non linear behavior of the material as well as for the presence of microstructure and dislocations. A way of doing that consists in the inclusion of one or more characteristic lengths within the framework of elastic-plastic rate constitutive relations. These lengths are typically of the same order of the compositional grain size, namely few microns, for polycrystalline metals. Several strain gradient (SG) plasticity models [3-9] based on phenomenological theories have been proposed for this purpose. They proved to be able to predict the size effect exhibited at the micron scale by ductile materials when subject to inhomogeneous plastic deformation, providing accurate predictions in the modeling of torsion of thin wires and bending of thin beams [5]. In a first attempt, Fleck and Hutchinson [4] presented a couple stress (CS) flow-theory of plasticity which involves strain rotation gradients only. This model includes an intrinsic characteristic material length $\ell$, which specifies the range where strain gradients are dominant. A further elastic length scale $\ell_{\mathrm{e}}$ was artificially introduced in order to partition the curvature rate tensor into elastic and plastic contributions. A generalization of this model was presented in [5], where the micro-rotation field is treated as an independent kinematical quantity with no direct dependence upon the displacement field. A flow-theory version of CS plasticity based on the indeterminate theory of CS elasticity [10] was also developed in [6, 7]. This model involves two material characteristic lengths, namely $\ell$ and $\ell^{\prime}$, whose effects on the ductile crack propagation are almost unexplored.

The asymptotic and full-field solutions for a stationary crack under Mode I and Mode II, obtained in [11-13] by using the CS deformation theory of plasticity presented in [4], revealed that the stress field dominates the couple stress field and thus the tractions level ahead of the crack-tip does not increase substantially. Therefore, rotation gradients seem to play a relatively small role in altering the stresses at the tip of a Mode I stationary crack. They are expected to have a major effect on the tractions ahead of a Mode III crack tip, whereas Mode I crack-tip fields are more significantly influenced by stretch gradients. It must be observed that the CS models failed to estimate the results of tests where also stretch gradients come into play, as for instance micro-indentation and void growth, since the strain gradients considered by these models derive uniquely from 
rotation gradients. Enhanced SG theories $[8,9,14]$ have been proposed to supply this lack. They may incorporate several material length scales and provide a link between the mesoscale SG plasticity level and the microscale dislocation mechanics. However, investigations of the stationary crack tip fields performed by adopting these refined constitutive models give physically unacceptable results. In particular, Chen et al. [15] found that the normal stresses ahead of the crack-tip under Mode I loading condition predicted by the SG theory of plasticity turn out to be compressive, while Shi et al. [16] showed that the crack-tip fields obtained by using the mechanism-based strain gradients (MSG) theory of plasticity do not admit a separable-variable form. Recent analyses carried out to investigate the effects of microstructure on crack propagation in ductile materials showed that the incorporation of couple stress and rotation gradients in the constitutive description, by using the flow theories of CS or SG plasticity, considerably improves the estimation of the tractions level ahead of the crack-tip and may explain the occurring of cleavage or atomic decohesion during the crack growth process, experimentally observed in ductile metals also [1]. In particular, the analyses of a steadily propagating crack-tip under Mode I loading conditions [17, 18] found that the couple stress field is dominant near to the crack-tip and produces a remarkable increase in the stress singularity, even for small strain hardening, whereas former investigations of crack propagation performed by using classical elastic-plastic constitutive models displaying linear strain hardening [19, 20], predicted an extremely weak stress singularity for the small values of the strain hardening coefficient generally adopted for ductile metals. Moreover, the investigations [17, 18] showed that the contribution of the elastic strain gradients strongly affects the asymptotic crack-tip fields, through the artificial elastic length scale $\ell_{\mathrm{e}}$.

The problem of Mode III crack propagation in ductile materials was first analyzed within the classical $\mathrm{J}_{2}$-flow theory for linear and isotropic hardening $[19,20]$ and extended to mixed isotropic/kinematic hardening [21]. These investigations found that the strength of the stress singularity turns out to be extremely weak for small strain hardening. The effects of strain gradients on the stress concentration at the tip of a Mode III stationary crack in a linear elastic material were considered in [22-25]. While strain gradients are introduced in $[22,25]$ through the second gradient of displacement, only rotation gradients are considered in $[23,24]$ by adopting a couple stress theory which account for a single characteristic length. The results obtained in [23] for the indeterminate theory of CS elasticity indicate that the skew-symmetric stress components have $r^{-3 / 2}$ singularity near the crack-tip, where $r$ is the distance to the crack tip, with no need to take stretch gradients into consideration. Although this singularity is much stronger than the conventional square-root singularity, it does not violate the boundness of strain energy surrounding the crack tip and leads to a finite energy release rate. The effects of strain rotation gradients on Mode III crack propagation in ductile metals was recently investigated in [26] by using the flow theory version of CS plasticity developed in [4]. The asymptotic analysis of the crack-tip fields performed in [26] showed that the contribution of the elastic strain gradients, which are related to the elastic length scale $\ell_{\mathrm{e}}$, strongly affects the asymptotic crack-tip fields, according to the results obtained for Mode I crack propagation [17]. Moreover, the skew-symmetric stress field asymptotically dominates the symmetric stress and couple stress fields, displaying a much stronger singularity than that predicted by the classical $\mathrm{J}_{2}$-flow theory. In particular, a strong increase in the singularity is observed if the elastic strain gradients are kept sufficiently small, namely for $\ell_{\mathrm{e}} \ll<\ell$.

In the present work, the effects of strain rotation gradients on ductile steady-state crack propagation under Mode III loading conditions are investigated by performing an asymptotic analysis of the crack-tip fields derived from the flow theory of the indeterminate CS plasticity with two characteristic material lengths of the same order, namely $\ell$ and $\ell^{\prime}$. Rotation gradients are found to give a significant contribution at a distance from a crack-tip smaller with respect to these characteristic lengths, whereas they become negligible at larger distances, with a gradual transition in the intermediate region. According to the results obtained in $[23,26]$ for a single characteristic material length $\ell$, the skew-symmetric stress field dominates the asymptotic field, producing thus a remarkable increase of tractions level at the crack tip, almost independently of the value of the strain hardening coefficient. The roles of both characteristic lengths are examined in detail and the influence of their ratio on the crack tip fields is numerically explored. The inclusion of two distinct characteristic lengths provides more realistic predictions on the tractions level ahead of the propagating crack-tip then the classical solution obtained for the $\mathrm{J}_{2}$-flow theory and gives more accurate results then the CS theory of plasticity with a single characteristic length, allowing the detailed mechanisms by which fracture may grow and propagate in ductile metals to be understood in more depth, up to the micron scale.

\section{Governing Equations}

The flow-theory version of the couple stress strain gradient plasticity developed in $[6,7]$ is adopted in the present study. This constitutive model derives from the indeterminate theory of CS elasticity developed by Koiter [10] and assumes the following kinematical compatibility conditions between the displacement vector $\mathbf{u}$, rotation vector $\boldsymbol{\theta}$, strain tensor $\boldsymbol{\varepsilon}$ and deformation curvature tensor $\chi$

$$
\boldsymbol{\varepsilon}=\frac{1}{2}\left(\nabla \mathbf{u}+\nabla \mathbf{u}^{\top}\right), \quad \boldsymbol{\theta}=\frac{1}{2} \operatorname{curl} \mathbf{u}, \quad \chi=\nabla \boldsymbol{\theta}, \quad \chi=\text { curl } \boldsymbol{\varepsilon} .
$$

Therefore, rotations are derived from displacements and the tensor field $\chi$ turns out to be irrotational. According to the CS theory [10] the non-symmetrical Cauchy stress tensor $\mathbf{t}$ can be decomposed into a symmetric part $\boldsymbol{\sigma}$ and a skew-symmetric part $\tau$, namely $t=\sigma+\tau$. In addition, the couple stress tensor $\boldsymbol{\mu}$ is introduced as the work-conjugated quantity of $\chi^{\top}$. 
The reduced surface tractions vector $\mathbf{p}$ and couple stress tractions vector $\mathbf{q}$ are defined respectively as

$$
\mathbf{p}=\mathbf{t}^{\top} \mathbf{n}+\frac{1}{2} \nabla \mu_{n n} \times \mathbf{n}, \quad \mathbf{q}=\boldsymbol{\mu}^{\top} \mathbf{n}-\mu_{n n} \mathbf{n},
$$

where $\mathbf{n}$ denotes the outward unit normal. The conditions of quasistatic equilibrium of forces and moments write

$$
\operatorname{div} \mathbf{t}^{\top}=\mathbf{0}, \quad \operatorname{div} \boldsymbol{\mu}^{\top}+\mathrm{e} \boldsymbol{\tau}=\mathbf{0} .
$$

where $\mathbb{e}$ is the third order alternator tensor. Within the context of small deformations incremental theory, the total strain rate $\dot{\boldsymbol{\varepsilon}}$ is the sum of elastic $\dot{\boldsymbol{\varepsilon}}^{\mathrm{e}}$ and plastic $\dot{\boldsymbol{\varepsilon}}^{\mathrm{p}}$ parts. Similarly, the total deformation curvature rate $\dot{\chi}$ is the sum of elastic $\dot{\chi}^{\mathrm{e}}$ and plastic $\dot{\chi}^{p}$ contributions. Both elastic parts are related to stress and couple stress rates through the following isotropic rate constitutive relations

$$
\dot{\boldsymbol{\varepsilon}}^{\mathrm{e}}=\frac{1}{2 G}\left[\dot{\boldsymbol{\sigma}}-\frac{v}{1+v}(\operatorname{tr} \dot{\boldsymbol{\sigma}}) \mathrm{I}\right], \quad \dot{\chi}^{\mathrm{eT}}=\frac{\dot{\boldsymbol{\mu}}-\eta \dot{\boldsymbol{\mu}}^{\mathrm{T}}}{2 G \ell^{2}\left(1-\eta^{2}\right)},
$$

where $G$ is the elastic shear modulus, $v$ the Poisson ratio and $\eta=\ell^{\prime} / \ell$ the dimensionless ratio between characteristic lengths introduced by Koiter [10], with $-1<\eta<1$. Both material characteristic lengths are related to the microstructure and generally are of the order of few microns for ductile polycrystalline metals. It is worth noting that the constitutive equations of the indeterminate CS theory do not define the skew-symmetric part $\tau$ of the total stress tensor $t$, which can be however obtained from the equilibrium equation (3) 2 . Moreover, within the couple stress theory $\boldsymbol{\chi}, \chi^{\mathrm{e}}, \chi^{\mathrm{p}}$ and $\boldsymbol{\mu}$ are purely deviatoric tensors.

The fundamental relationships of the constitutive model are briefly summarized below.

- $\quad$ Yield condition

$$
f(\Sigma, Y)=\Sigma-Y=0
$$

where

$Y=$ uniaxial flow stress for isotropic hardening

$$
\begin{aligned}
& \Sigma=\sqrt{\frac{3}{2}\left(\mathbf{S} \cdot \mathbf{S}+\frac{\boldsymbol{\mu} \cdot \boldsymbol{\mu}-\eta \boldsymbol{\mu} \cdot \boldsymbol{\mu}^{\top}}{\ell^{2}\left(1-\eta^{2}\right)}\right)}=\text { overall effective stress } \\
& \mathbf{S}=\text { deviatoric part of the symmetric stress tensor } \boldsymbol{\sigma} \text {. }
\end{aligned}
$$

- $\quad$ Associative flow rules

$$
\dot{\boldsymbol{\varepsilon}}^{\mathrm{p}}=\Lambda \frac{\partial f}{\partial \boldsymbol{\sigma}}=\frac{3 \Lambda}{2 \Sigma} \mathbf{S}, \quad \dot{\boldsymbol{\chi}}^{\mathrm{p} \top}=\Lambda \frac{\partial f}{\partial \boldsymbol{\mu}}=\frac{3 \Lambda}{2 \Sigma} \frac{\left(\boldsymbol{\mu}-\eta \boldsymbol{\mu}^{\top}\right)}{\ell^{2}\left(1-\eta^{2}\right)}
$$

$$
\Lambda=\text { plastic multiplier }
$$

- $\quad$ Linear isotropic hardening rule

$$
\dot{Y}=\Lambda H
$$

where $H$ is the constant hardening modulus, which depends on the ratio $\alpha=G_{t} / G$ between the tangent plastic shear modulus $G_{t}$ and the elastic shear modulus $G(0<\alpha<1)$, namely

$$
H=\frac{3 \alpha}{1-\alpha} G \text {. }
$$

- Consistency condition

$$
\dot{f}=0, \quad \Rightarrow \quad \dot{\Sigma}=\dot{Y},
$$

which gives the non-negative plastic multiplier $\Lambda$ as

$$
\Lambda=\left\{\begin{array}{cll}
\langle\dot{\Sigma}\rangle / H & \text { if } & f(\Sigma, \mathrm{Y})=0 \\
0 & \text { if } & f(\Sigma, \mathrm{Y})<0,
\end{array} \quad \dot{\Sigma}=\frac{3}{2 \Sigma}\left[\mathbf{S} \cdot \dot{\mathbf{S}}+\frac{\left(\boldsymbol{\mu}-\eta \boldsymbol{\mu}^{\top}\right) \cdot \dot{\boldsymbol{\mu}}}{\ell^{2}\left(1-\eta^{2}\right)}\right] .\right.
$$

The elastic-plastic rate constitutive equations resulting from (4) and (7) are

$$
\dot{\boldsymbol{\varepsilon}}=\frac{1}{2 G}\left[\dot{\boldsymbol{\sigma}}-\frac{v}{1+v}(\operatorname{tr} \dot{\boldsymbol{\sigma}}) \mathbf{l}\right]+\frac{3 \Lambda}{2 \Sigma} \mathbf{S}, \quad \ell^{2} \dot{\chi}^{\top}=\frac{1}{1-\eta^{2}}\left[\frac{1}{2 G}\left(\dot{\boldsymbol{\mu}}-\eta \dot{\boldsymbol{\mu}}^{\top}\right)+\frac{3 \Lambda}{2 \Sigma}\left(\boldsymbol{\mu}-\eta \boldsymbol{\mu}^{\top}\right)\right] .
$$


These equations hold when the stress and couple stress fields obey the yield condition (5). Otherwise, the plastic multiplier $\Lambda$ is set equal to 0 . In this case, isotropic elastic behavior with couple stress is recovered. Therefore, strain gradient effects also occur for a purely elastic response. Finally, note that constitutive equations (12) reduce to the widely used $\mathrm{J}_{2}$-flow theory of plasticity when the strain gradients are vanishing small.

\section{Mode III Crack Propagation Problem}

The problem of a semi-infinite plane crack propagating at constant velocity $V$ along a rectilinear path is considered in the present section by performing an asymptotic analysis of the crack tip fields. Reference is made to a cylindrical coordinate system $\left(r, \theta, x_{3}\right)$ centred at the crack-tip and moving with it towards the $\theta=0$ direction, where the $x_{3}$-axis coincides with the straight crack front. The condition of steady-state propagation yields the following time derivative rule for any arbitrary scalar function $\phi$ :

$$
\dot{\phi}=\frac{V}{r}\left(\frac{\partial \phi}{\partial \theta} \sin \theta-r \frac{\partial \phi}{\partial r} \cos \theta\right)
$$

For antiplane problems, the non-vanishing stress and couple stress components with respect to a cylindrical coordinate system in cylindrical coordinates are $\sigma_{r 3}, \sigma_{\theta 3}, \tau_{r 3}, \tau_{\theta 3}, \mu_{r r}, \mu_{r \theta}, \mu_{\theta r}$ and $\mu_{\theta \theta}$, where $\mu_{\theta \theta}=-\mu_{r r}$. Accordingly, the strain and deformation curvature components are $\varepsilon_{r 3}, \varepsilon_{\theta 3}, \chi_{\theta r}, \chi_{r \theta}, \chi_{r r}$, and $\chi_{\theta \theta}$, where $\chi_{\theta \theta}=-\chi_{r r}$. The equilibrium conditions (5) write

$$
\left(r t_{r 3}\right)_{, r}+t_{\theta 3, \theta}=0, \quad\left(r \mu_{r r}\right)_{, r}+\mu_{\theta r, \theta}-\mu_{\theta \theta}+2 r \tau_{\theta 3}=0, \quad\left(r \mu_{r \theta}\right)_{, r}+\mu_{\theta \theta, \theta}+\mu_{\theta r}-2 r \tau_{r 3}=0 .
$$

The kinematical compatibility conditions (1) between displacement, strains and deformation curvatures imply in rate form

$$
\begin{array}{lll}
v_{3}=\dot{u}_{3} & v_{3, r}=2 \dot{\varepsilon}_{r 3}, & v_{3, \theta}=2 r \dot{\varepsilon}_{\theta 3}, \\
\dot{\chi}_{r r}=\dot{\varepsilon}_{3 \theta, r}, & \dot{\chi}_{r \theta}=\dot{\varepsilon}_{3 \theta, \theta}+\dot{\varepsilon}_{3 r} / r, & \dot{\chi}_{\theta r}=-\dot{\varepsilon}_{3 r, r .} .
\end{array}
$$

Equations (14)-(16) together with rate constitutive equations (8) and (12) form a system of first order PDEs that governs the problem of the crack propagation. The asymptotic crack-tip fields are sought in the separable variable form $\phi(r, \theta)=r^{p} F(\theta)$, where the exponent $p$ defines the radial dependence of the generic function $\phi$ as $r \rightarrow 0$. A qualitative analysis performed in [26] gives the relative order of singularity of the crack-tip fields, which holds in the present context also. Therefore, the displacement, velocity, stress and couple stress asymptotic crack-tip fields are assumed in the form

$$
\begin{aligned}
& u_{3}(r, \theta)=r\left(\frac{r}{R}\right)^{p} u(\theta), \quad v_{3}(r, \theta)=v\left(\frac{r}{R}\right)^{p} w(\theta), \\
& \sigma_{\alpha 3}(r, \theta)=G\left(\frac{r}{R}\right)^{p} s_{\alpha}(\theta), \quad \mu_{\alpha \beta}(r, \theta)=G \frac{\ell^{2}}{r}\left(\frac{r}{R}\right)^{p} M_{\alpha \beta}(\theta), \quad \tau_{\alpha 3}(r, \theta)=G \frac{\ell^{2}}{r^{2}}\left(\frac{r}{R}\right)^{p} t_{\alpha}(\theta),
\end{aligned}
$$

where the Greek indices $\alpha$ and $\beta$ stand for polar coordinates $r$ and $\theta$. Since $\boldsymbol{\mu}$ and $\mathbf{M}$ are purely deviatoric tensors, the condition $M_{\theta \theta}=-M_{r r}$ holds true. As $r$ goes to zero, the symmetric stress, couple stress and skew symmetric stress fields (18) behave as $r^{p}, r^{p-1}$ and $r^{p-2}$, respectively. Therefore, the latter field gives the most singular contribution near to the crack tip, for $r<\ell$. Oppositely, for $r>\ell$, the couple stress and skew symmetric stress fields become negligible with respect to the symmetric stress field, in agreement with the classical theories of elastic-plastic fracture mechanics. The constant $R$ in (17) and (18) plays the role of an undetermined amplitude factor for the leading order asymptotic fields. The solution of the homogeneous asymptotic problem can indeed be determined up to an amplitude factor, which depends on far-field loading and specimen geometry and can be estimated by matching the asymptotic solution with the far-field conditions. Nevertheless, the asymptotic analysis can capture the strength of the singularity of the crack tip field and their angular variation, once a normalization condition is adopted.

According to the asymptotic representations (18) of the stress and couple stress fields the overall effective stress and flow stress fields near the crack-tip may be assumed in the form

$$
\Sigma(r, \theta)=G \frac{\ell}{r}\left(\frac{r}{R}\right)^{p} \Gamma(\theta), \quad Y(r, \theta)=G \frac{\ell}{r}\left(\frac{r}{R}\right)^{p} \gamma(\theta),
$$

where the function $\Gamma$ resulting from (6) and (18) is

$$
\Gamma=\sqrt{\frac{3}{1-\eta^{2}}\left[(1-\eta) M_{r r}^{2}+\frac{M_{r \theta}^{2}+M_{\theta r}^{2}}{2}-\eta M_{r \theta} M_{\theta r}\right]},
$$


since near the crack-tip, namely for $r<\ell$, the couple stress field gives the most singular contribution to the effective stress and, thus, to the yield condition. When the asymptotic fields (18) are introduced into equilibrium equations (14) the following ODEs are derived at leading order

$$
t_{\theta}^{\prime}=(1-p) t_{r}, \quad M_{\theta r}^{\prime}=-(1+p) M_{r r}-2 t_{\theta}, \quad M_{r r}{ }^{\prime}=M_{\theta r}+p M_{r \theta}-2 t_{r}
$$

where ()$^{\prime}=d() / d \theta$. By using the steady-state derivative rule (13), the rates of $\sigma, \mu$ and $Y$ in (18) and (19) can be written in the form

$$
\dot{\sigma}_{\alpha 3}(r, \theta)=G \frac{V}{r}\left(\frac{r}{R}\right)^{p} h_{\alpha}(\theta), \quad \quad \dot{\mu}_{\alpha \beta}(r, \theta)=G V \frac{\ell^{2}}{r^{2}}\left(\frac{r}{R}\right)^{p} H_{\alpha \beta}(\theta), \quad \dot{Y}(r, \theta)=G V \frac{\ell}{r^{2}}\left(\frac{r}{R}\right)^{p} \kappa(\theta),
$$

where the polar components $h_{\alpha}$ and $H_{\alpha \beta}$ and the function $\kappa$ follow from (18) $)_{1,2}$ and $(19)_{2}$ as

$$
\begin{array}{ll}
h_{r}=\left(s_{r}^{\prime}-s_{\theta}\right) \sin \theta-p s_{r} \cos \theta, & h_{\theta}=\left(s_{\theta}{ }^{\prime}+s_{r}\right) \sin \theta-p s_{\theta} \cos \theta, \\
H_{r r}=\left(M_{r r}{ }^{\prime}-M_{\theta r}-M_{r \theta}\right) \sin \theta+(1-p) M_{r r} \cos \theta=-H_{\theta \theta}, & H_{r \theta}=\left(M_{r \theta}{ }^{\prime}+2 M_{r r}\right) \sin \theta+(1-p) M_{r \theta} \cos \theta, \\
H_{\theta r}=(1-p)\left(M_{r r} \sin \theta+M_{\theta r} \cos \theta\right)-2 t_{\theta} \sin \theta . & \kappa=\gamma^{\prime} \sin \theta+(1-p) \gamma \cos \theta .
\end{array}
$$

Note that the equilibrium condition $(21)_{2}$ has been used in $(25)_{1}$. The rate constitutive relations (12) require that the strain and deformation curvature rates must have the same radial dependence assumed for the stress and couple stress rates in $(22)_{1,2}$, namely

$$
\dot{\varepsilon}_{\alpha 3}(r, \theta)=\frac{V}{r}\left(\frac{r}{R}\right)^{p} d_{\alpha}(\theta), \quad \dot{\chi}_{\alpha \beta}(r, \theta)=\frac{V}{r^{2}}\left(\frac{r}{R}\right)^{p} X_{\alpha \beta}(\theta) .
$$

In contrast with the problems of Mode I and Mode II crack [11, 12, 17], the dominant strain rate field for the antiplane crack problem turns out to be rotational. Therefore, the compatibility equations (16) admit non-vanishing deformation curvature rates at lowest order. In agreement with the asymptotic representations (17) and (26), the kinematical compatibility conditions (15) 2,3 and (16) can be written as

$$
d_{r}=\frac{1}{2} p w, \quad w^{\prime}=2 d_{\theta} . \quad X_{r r}=(p-1) d_{\theta}, \quad X_{\theta r}=\frac{1}{2}(1-p) p w \quad X_{r \theta}=d_{\theta}{ }^{\prime}+\frac{1}{2} p w .
$$

After the definitions (19) of the effective and flow stress fields, the yield condition (5) writes $\Gamma=\gamma$. The introduction of the asymptotic fields (17)-(19) and their rates (22) and (26) in the incremental constitutive relationships (12) and (8), by using $(27)_{1,5}$, yields the following set of equations

$$
\begin{array}{ll}
h_{r}+2\langle\lambda\rangle s_{r}=p w, & h_{\theta}+2\langle\lambda\rangle s_{\theta}=2 d_{\theta} . \\
H_{r r}+2\langle\lambda\rangle M_{r r}=2(1+\eta) X_{r r}, & \left(H_{r \theta}-\eta H_{\theta r}\right)+2\langle\lambda\rangle\left(M_{r \theta}-\eta M_{\theta r}\right)=2\left(1-\eta^{2}\right) X_{\theta r}, \\
\left(H_{\theta r}-\eta H_{r \theta}\right)+2\langle\lambda\rangle\left(M_{\theta r}-\eta M_{r \theta}\right)=\left(1-\eta^{2}\right)\left(2 d_{\theta}^{\prime}+p w\right), & 3 \kappa=2\langle\lambda\rangle h \Gamma,
\end{array}
$$

where $h=H / G$ is the non-dimensional hardening modulus and the function $\lambda(\theta)$ is defined such that $\lambda=0$ when $\Gamma<\gamma$, and

$$
\lambda=\frac{1}{h^{*}}\left[2(1-\eta) M_{r r} H_{r r}+\left(M_{r \theta}-\eta M_{\theta r}\right) H_{r \theta}+\left(M_{\theta r}-\eta M_{r \theta}\right) H_{\theta r}\right]
$$

when $\Gamma=\gamma$, in agreement with (11), being $h^{*}=h\left(1-\eta^{2}\right)(2 \Gamma / 3)^{2}$. Equations (28)-(30) hold true when the effective stress and flow stress obey the yield condition. Under elastic unloading or neutral loading $\lambda \leq 0$ and thus equations (28)-(30) reduce to the rate constitutive relations of linear isotropic elasticity with strain gradient effects.

\section{Explicit Form of the ODEs System}

An explicit form of the ODEs system is now derived by rearranging the equations presented in the previous subsection. The introduction of (31) in equations (29) after some algebraic manipulations, allows to obtain the following explicit expression for the functions $H_{r r}$ and $H_{r \theta}$ :

where

$$
\left[\begin{array}{l}
H_{r r} \\
H_{r \theta}
\end{array}\right]=\frac{1}{\Delta}\left[\begin{array}{cc}
h^{*}+2\left(M_{r \theta}-\eta M_{\theta r}\right)^{2} & -2 M_{r r}\left(M_{r \theta}-\eta M_{\theta r}\right) \\
-4(1-\eta) M_{r r}\left(M_{r \theta}-\eta M_{\theta r}\right) & h^{*}+4(1-\eta) M_{r r}^{2}
\end{array}\right]\left[\begin{array}{c}
z_{r} \\
z_{\theta}
\end{array}\right],
$$

$$
\begin{gathered}
\Delta=h^{*}\left[h^{*}+2\left(M_{r \theta}-\eta M_{\theta r}\right)^{2}+4(1-\eta) M_{r r}{ }^{2}\right], \\
z_{r}=2 h^{*}(1+\eta) X_{r r}-2 M_{r r}\left(M_{\theta r}-\eta M_{r \theta}\right) H_{\theta r}, \quad z_{\theta}=2 h^{*}\left(1-\eta^{2}\right) X_{\theta r}+\left[h^{*} \eta-2\left(M_{r \theta}-\eta M_{\theta r}\right)\left(M_{\theta r}-\eta M_{r \theta}\right)\right] H_{\theta r} .
\end{gathered}
$$


Note that $\Delta$ is always positive for $h>0$, being $-1<\eta<1$. Since the derivatives of the unknown angular functions do not enter the algebraic expressions (32), (25) 1 and (27) $)_{1,2}$ for $H_{r r}, H_{r \theta}, H_{\theta r}, X_{r r}$ and $X_{\theta r}$, the expressions (32) and (25) $)_{1}$ for $H_{r r}, H_{r \theta}$ and $H_{\theta r}$ can be introduced in (31) to obtain $\lambda$ in explicit form. Moreover, the derivatives with respect to $\theta$ of the stress and couple stress components $s_{r}, s_{\theta} M_{r r}$ and $M_{r \theta}$ follow from (23) and (24) as

$$
\begin{array}{ll}
s_{r}{ }^{\prime}=s_{\theta}+\left(h_{r}+p s_{r} \cos \theta\right) / \sin \theta, & M_{r r}{ }^{\prime}=M_{\theta r}+M_{r \theta}-(1-p) M_{r r} \cot \theta+H_{r r} / \sin \theta, \\
s_{\theta}{ }^{\prime}=-s_{r}+\left(h_{\theta}+p s_{\theta} \cos \theta\right) / \sin \theta, & M_{r \theta}{ }^{\prime}=-2 M_{r r}-(1-p) M_{r \theta} \cot \theta+H_{r \theta} / \sin \theta .
\end{array}
$$

where $h_{r}, h_{\theta}, H_{r r}$ and $H_{r \theta}$ are given by (28) and (32). Similarly, from (25) 2 and (30) 2 and by using the steady-state condition (13) for the derivative of the displacement field $(17)_{1}$ one obtains

$$
\gamma^{\prime}=(p-1) \gamma \cot \theta+\frac{2}{3}\langle\lambda\rangle h \Gamma / \sin \theta, \quad \quad u^{\prime}=[w+(p+1) u \cos \theta] / \sin \theta .
$$

Equations $(21)_{1,2},(27)_{2},(30)_{1},(33)$-(35) form a system of 10 first order homogeneous ODEs governing the near-tip stress and velocity fields for the antiplane crack propagation problem in couple stress strain gradient solids. This system may be written in the following explicit form:

$$
\mathbf{y}^{\prime}(\theta)=\left\{\begin{array}{lll}
\mathbf{f}_{\mathrm{p}}(\theta, \mathbf{y}(\theta)) & \text { if } \quad \Gamma=\gamma \quad \text { and } \lambda>0, \\
\mathbf{f}_{\mathrm{e}}(\theta, \mathbf{y}(\theta)) & \text { if } \quad \Gamma<\gamma \quad \text { or } \quad(\Gamma=\gamma \quad \text { and } \quad \lambda \leq 0),
\end{array}\right.
$$

where the vector $\mathbf{y}=\left\{w, t_{\theta}, s_{r}, s_{\theta}, M_{r r}, M_{r \theta}, M_{\theta r}, d_{\theta}, \gamma, u\right\}$ collects the 10 unknown functions. Note that $t_{r}$ and $d_{r}$ are not primary unknown functions because the former may be obtained from $(21)_{3}$, by using $(33)_{2}$, whereas the latter is given by the algebraic equation $(27)_{1}$ once all the functions in $y$ are known. The unknown exponent $p$ can be determined as an eigenvalue of the nonlinear homogeneous problem (36), once a normalization condition is assumed for the asymptotic solution.

The flow-theory of CS plasticity adopted in the present work allows to consider the occurring of elastic unloading. The position of a material point moving along a rectilinear path with respect to the crack-tip is specified by the angular coordinate $\theta$. A generic material point near the trajectory of the crack-tip experiences plastic loading ahead of the crack-tip. Depending on the values of the material parameters, it may undergo elastic unloading at $\theta=\theta_{1}$ and subsequent plastic reloading at $\theta=\theta_{2}$ or, alternatively, the zone surrounding the crack tip may be fully plastic. The condition of elastic unloading may occur at the angle $\theta_{1}$ defined by the condition $\dot{\Sigma}=0$, so that $\lambda\left(\theta_{1}\right)=0$. Then, plastic reloading may occurs at crack flanks at the angular coordinate $\theta_{2}$ where the material point reaches a stress state obeying the yield condition discarded at unloading.

\section{Mode III Boundary Conditions}

The symmetry condition of Mode III crack propagation restricts the analysis to the range $0 \leq \theta \leq \pi$. It is reasonable to assume regular behavior of the angular functions at $\theta=0$, so that relations (23)-(25) imply

$$
h_{\alpha}(0)=-p s_{\alpha}(0), \quad \quad H_{\alpha \beta}(0)=(1-p) M_{\alpha \beta}(0), \quad \kappa(0)=-p \gamma(0) .
$$

By using condition (37) 2 , the function $\lambda$ defined in (29) at $\theta=0$ attains the initial value

$$
\lambda(0)=\frac{3}{2 h}(1-p),
$$

which is positive for $p<1$. The skew-symmetry of the antiplane crack problem requires ahead of the crack-tip at $\theta=0$ :

$$
u(0)=0, \quad w(0)=0, \quad M_{\theta r}(0)=0 .
$$

After some algebraic manipulations, the introduction of (37)-(39) into the constitutive equations (28)-(30) for $\theta=0$ yields

$$
s_{r}(0)=0, \quad d_{\theta}(0)=\frac{3-(3+h) p}{2 h} s_{\theta}(0), \quad M_{r r}(0)=(1+\eta)\left(p-\frac{3}{3+h}\right) s_{\theta}(0), \quad M_{r \theta}(0)=0, \quad d_{\theta}^{\prime}(0)=0 .
$$

Vanishing of reduced tractions $(2)_{1}$ along the radial direction at $\theta=0$ requires $M_{r r}{ }^{\prime}(0)=2 t_{r}(0)$. Hence, from the equilibrium condition $(21)_{3}$ it follows that

$$
t_{r}(0)=0, \quad M_{r r}^{\prime}(0)=0 .
$$

In order to solve the system (34), the Runge-Kutta procedure is used (subroutine DIVPRK of the IMSL library). This approach requires knowledge of the initial values $\mathbf{y}(0)$. Since all the assigned boundary conditions (39)-(43) are homogeneous, the normalization condition for the skew-symmetric stress

$$
t_{\theta}(0)=-1
$$

is adopted to avoid the trivial solution. The negative sign is considered since the results obtained in [26] reveal that the skew- 
symmetric shear stress $\tau_{\theta 3}$ ahead of the crack tip and the crack-tip opening displacement occur with opposite signs.

Under the assumption that the zone ahead of the crack-tip is experiencing plastic loading, the yield condition $\gamma(0)=\Gamma(0)$ holds true, where $\Gamma(0)$ may be obtained by introducing $(39)_{3},(40)_{4}$ in $(20)$, so that

$$
\gamma(0)=\sqrt{3 M_{r r}^{2}(0) /(1+\eta)}
$$

By taking the derivatives with respect to $\theta$ of $(23)_{1}$ and $(28)_{2}$, evaluated at $\theta=0$ and using $(27)_{2}$ and $(40)_{1,2}$, it follows that

$$
s_{r}^{\prime}(0)=\left(p+\frac{h}{3+h}\right) s_{\theta}(0), \quad s_{\theta}{ }^{\prime}(0)=0 .
$$

Moreover, from the equilibrium equations $(21)_{1,2}$ and the derivatives of $(24)_{2}$ and $(28)_{3}$ evaluated at $\theta=0$, by using $(40)_{4}$, the following results can be found

$$
t_{\theta}^{\prime}(0)=0, \quad M_{r \theta}{ }^{\prime}(0)=\frac{2(1-p)(1+\eta)[(2-p)(1+p)+2 \lambda(0)(p+\eta)]}{[1-p+2 \lambda(0)][2-p+2 \lambda(0)]} d_{\theta}(0)-2 \eta t_{\theta}(0) .
$$

By taking the derivative of $\Gamma(\theta)$ and $\lambda(\theta)$ from $(20)$ and $(29)$ and using $(39)_{3},(40)_{4}$, and $(41)_{2}$ it follows that $\Gamma^{\prime}(0)=\lambda^{\prime}(0)=0$. Then, from the derivatives of (35) evaluated at $\theta=0$ and the use of $(27)_{2}$ it follows that

$$
\gamma^{\prime}(0)=0, \quad u^{\prime}(0)=-2 d_{\theta}(0) / p .
$$

The conditions (39)-(43) give the initial values of all the unknown functions at $\theta=0$ depending on $p$ and $s_{\theta}(0)$. The values of $p$ and $s_{\theta}(0)$ can be calculated by an iterative procedure based on the achievement of the boundary conditions at $\theta=\pi$. Therein, the vanishing of the reduced tractions (2) on the crack surfaces implies the following two conditions

$$
2 t_{\theta}(\pi)+(1-p) M_{r r}(\pi)=0, \quad M_{\theta r}(\pi)=0 .
$$

This iteration is performed by using the modified Powell hybrid method (subroutine DNEQNF of the IMSL library), until the conditions (47) on the general tractions are verified within a prescribed accuracy.

It must be remarked that the numerical integration procedure of the governing ODE system (36) displays a numerical difficulty at $\theta=0$, due to the term $\sin \theta$ which multiplies the highest order derivative. This inconvenience can be bypassed by performing a Taylor series expansions of the unknown angular functions collected in the vector $\mathbf{y}$, starting at $\theta=0$, namely:

$$
\mathbf{y}(\varepsilon)=\mathbf{y}(0)+\varepsilon \mathbf{y}^{\prime}(0)+o(\varepsilon)
$$

where $\varepsilon<<1$. In this way, the unknown functions can be evaluated at $\theta=\varepsilon$, since the derivatives of the unknown functions at $\theta=0$ are known from relations $(21)_{1,2},(40)_{5},(41)_{2}$ and (44)-(46). Once that $\mathbf{y}(\varepsilon)$ has been determined as in (48) within an error lower than $\varepsilon$, the numerical integration of (36) can be performed by starting at $\theta=\varepsilon$, rather than at $\theta=0$.

\section{Results}

Figure 1 shows the variation of the exponent $p$ with the ratio $\eta=\ell^{\prime} \ell \ell$ for different values of the strain hardening coefficient $\alpha$. The ratio $\eta$ has a limited influence on $p$, since just a small variation of $p$ occurs as $\eta$ ranges between -1 and 1 . However, the ratio $\eta$ produces remarkable changes in the angular distribution of the crack tip fields. As can be expected, the larger is the coefficient $\alpha$, the lower is the exponent $p$ and thus the stronger is the singularity of the couple stress and skew symmetric stress fields, which behave as $r^{p-1}$ and $r^{p-2}$, respectively. Since the values assumed by $p$ range between 0.8 and 1 for almost every realistic value of the strain hardening coefficient for ductile metals, the hardening coefficient $\alpha$ has a small influence on the strength of stress singularity, unlike in classical plasticity $[19,20]$. Actually, the skew symmetric stress field displays a strong singularity almost independently of $\alpha$. It must be remarked that it does not contribute to the effective stress $\Sigma$ and to the strain-energy density, which are instead dominated by the weakly singular couple-stress field, so that the flux of energy toward the crack-tip remains finite. It follows that the tractions level ahead of the crack tip increases with respect to the classical $\mathrm{J}_{2}$ flow theory due to the contribution of the skew-symmetric stress components, also for very small strain hardening. In fact, the solution obtained for conventional elastic-plastic response predicts a weak stress singularity for small strain-hardening, which becomes vanishing small in the limit case of elastic-perfectly plastic behavior of the material, namely for $\alpha=0$.

Figures 2-3 display the angular distributions of the asymptotic crack-tip fields for $\alpha=0.01$ and $\eta= \pm 0.5$. In both cases the zone surrounding the crack-tip at distance lower than the characteristic material lengths is fully plastic. In particular, the angular variations of symmetric stresses (a), out-of-plane velocity and displacement (b), couple stresses (c) and skew symmetric stresses $(d)$ are plotted therein. All functions are normalized by condition (42). 


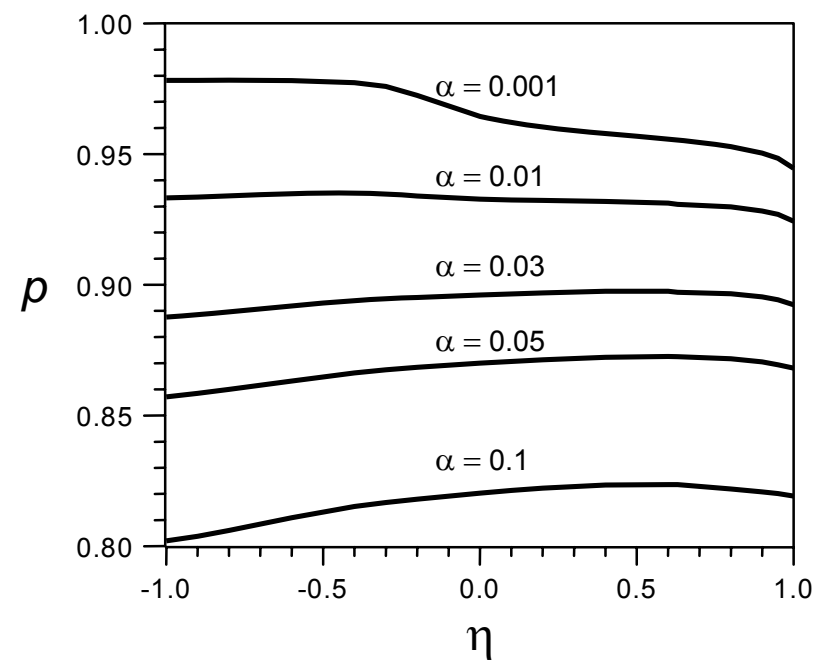

Figure 1. Variation of the exponent $p$ with the ratio $\eta=\ell^{\prime} / \ell$ for different values of the hardening coefficient $\alpha$.

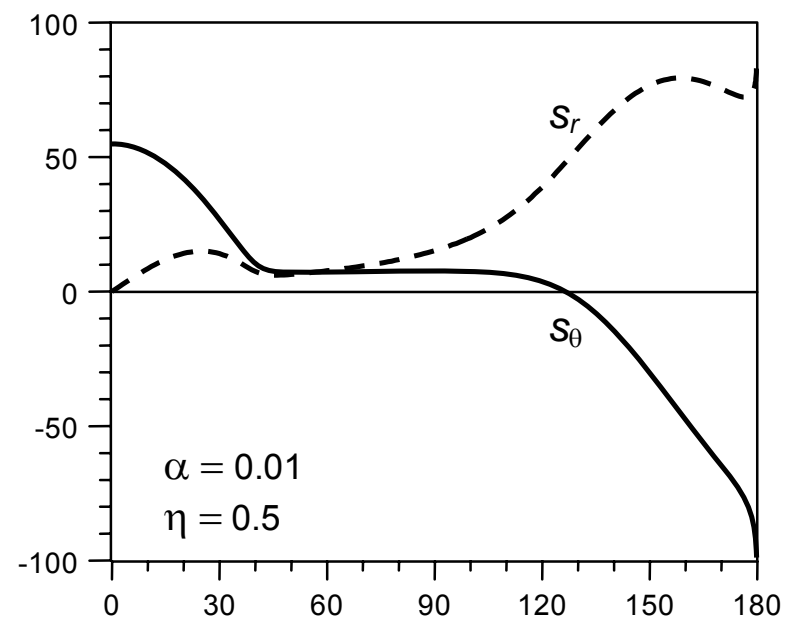

a)

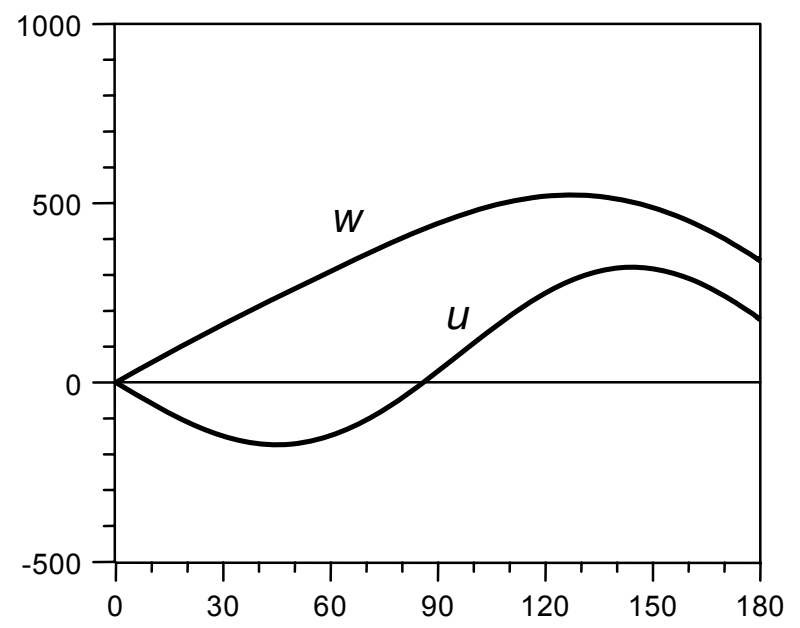

b)

$\theta$

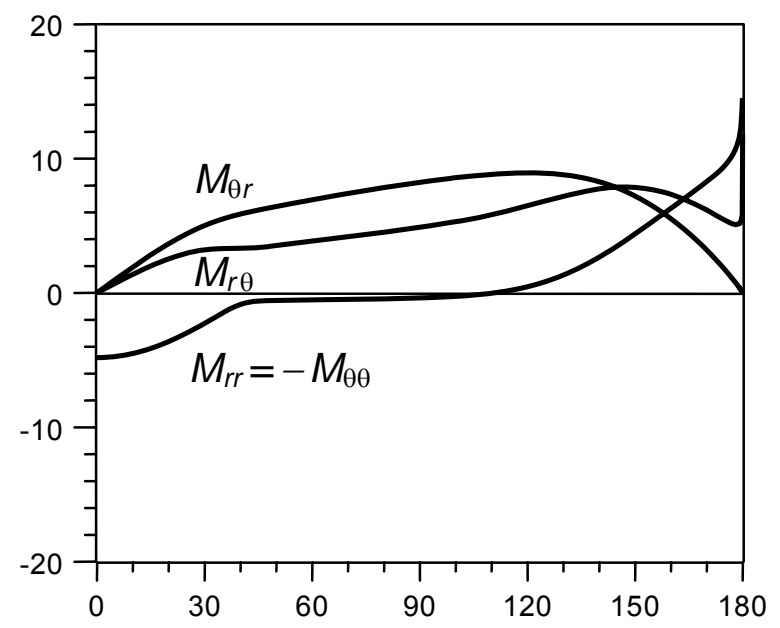

c)

$\theta$

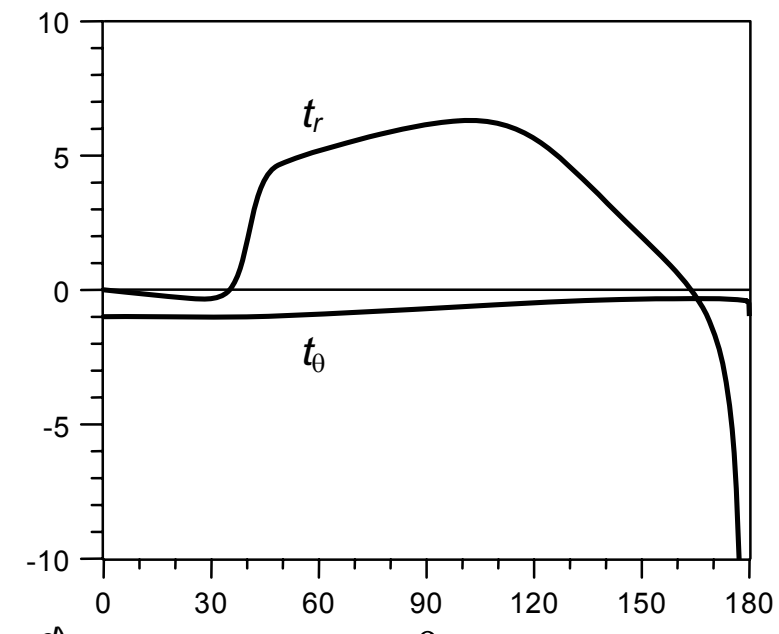

d) $\theta$

Figure 2. Angular variation of the crack-tip fields for $\alpha=0.01$ and $\eta=0.5$ 


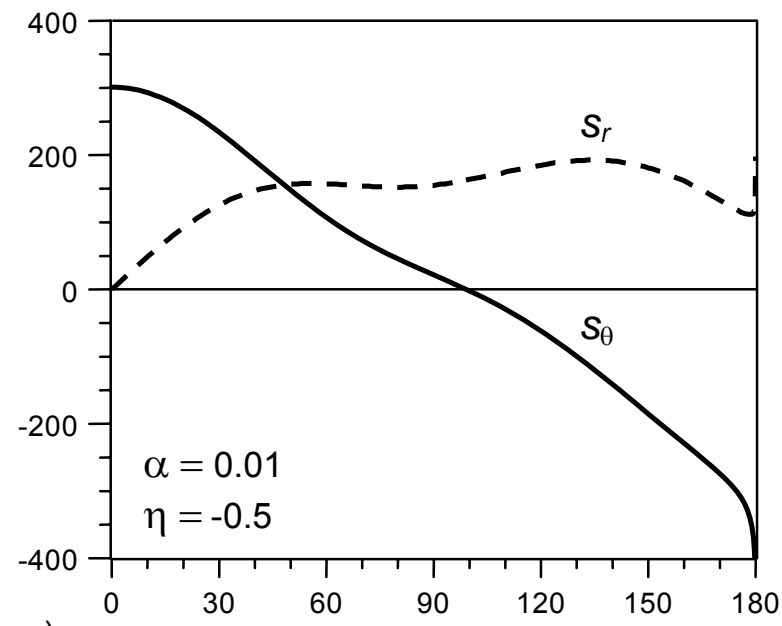

a)
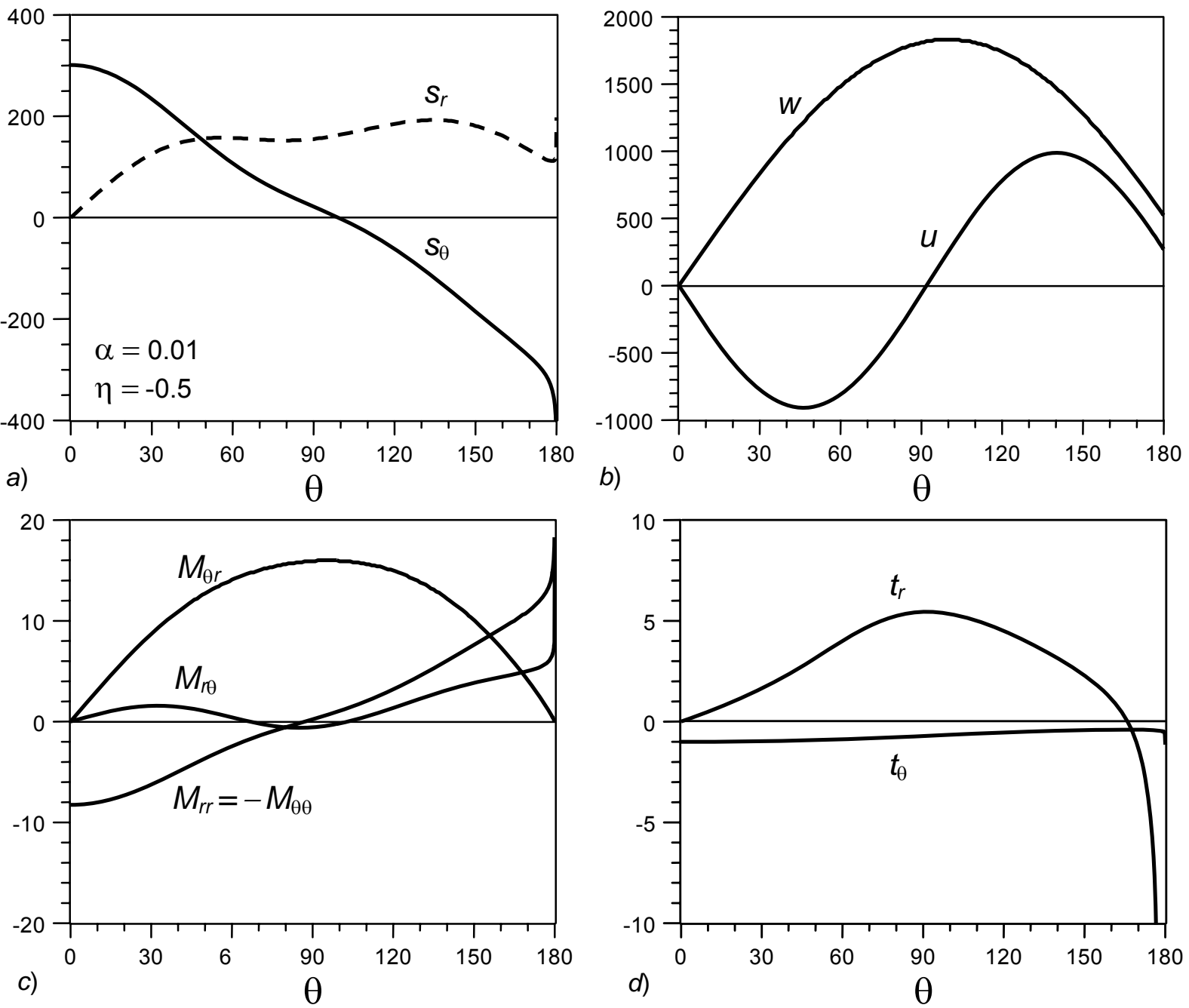

b)

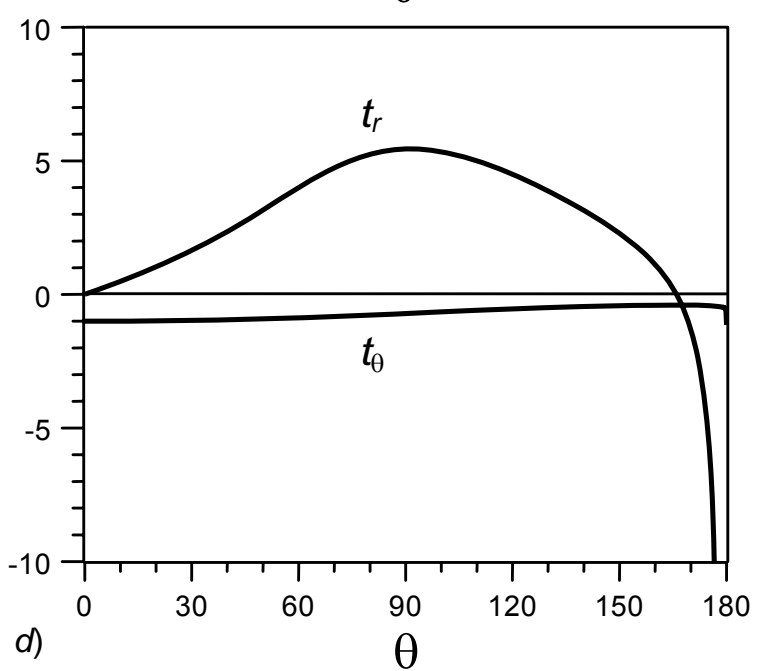

Figure 3. Angular variation of the crack-tip fields for $\alpha=0.01$ and $\eta=-0.5$

The curves plotted in Figures $2 b$ and $3 b$ show that the displacements ahead and behind the crack-tip are opposite in sign, unlike the classical Mode III crack-tip fields in non-polar materials [19-21]. Similar results have been also obtained for a Mode III crack both for CS elastic [23] and CS elastic-plastic material behavior [26]. This unusual aspect seems to be due to the presence of microstructures (compositional grains) introduced through the characteristic lengths $\ell$ and $\ell^{\prime}$. During crack growth the separation process between two material particles at the crack tip can be divided into two steps. In the first step the particles rotate with respect to each other. Only in the second step they move apart. The local rotation of grains and particles currently at the crack-tip produces opposite displacements ahead and behind the crack-tip under Mode III loading condition thus originating a scissors effect. These results confirm that the microstructure remarkably affect the solutions of fracture mechanics problems.

The angular variations of the skew-symmetric stress components are plotted in Figures $2 d$ and $3 d$. In agreement with the inversion of the displacement field ahead of the crack-tip, the shear stress $t_{\theta}$ is also negative therein and, thus, opposite to its counterpart in the classical Mode III solution. For positive values of the ratio $\eta$ the radial shear stress component $t_{r}$ also displays negative values in a small sector ahead of the crack-tip. This switch in the shear direction agrees with the findings in $[23,26]$.

\section{Conclusions}

The obtained results show that the use of the SG theory of plasticity with two characteristic lengths for the analysis of the stress field near the tip of a propagating Mode III crack gives accurate predictions on the increase of the tractions level ahead of the crack-tip occurring at very small distances from it, comparable with the size of the compositional grains. Moreover, the 
lower radius of validity for the obtained asymptotic solution is much smaller than that found for the classical theories of plasticity, allowing to understand the detailed mechanisms by which fracture may grow and propagate in ductile polycrystalline metals up to the micron scale.

\section{Acknowledgments}

Financial support from the Italian Ministry of Education, University and Research (MIUR) in the framework of the Project PRIN2004 "Fracture toughness and macroscopic mechanical properties of materials with microstructure" is gratefully acknowledged.

\section{References}

1. Elssner, G., Korn, D. and Ruehle, M., "The influence of interface impurities on fracture energy of UHV diffusion bonded metal-ceramic bicrystals". Scripta Metallurgica et Materialia, 31, 1037-1042 (1994).

2. Drugan, W.J., Rice, J.R. and Sham, T.L., "Asymptotic analysis of growing plane strain tensile cracks in elastic-ideally plastic solids". Journal of the Mechanics and Physics of Solids, 30, 447-473 (1982).

3. Hutchinson, J.W., "Plasticity at the micron scale". International Journal of Solids and Structures, 37, $225-238$ (2000).

4. Fleck, N.A. and Hutchinson, J.W., "A phenomenological theory for strain gradient effects in plasticity". Journal of the Mechanics and Physics of Solids, 41, 1825-1857 (1993).

5. Chen, S.H. and Wang, T.C., "A new deformation theory with strain gradient effects". International Journal of Plasticity, 18, 971-995 (2002).

6. Ristinmaa, M. and Vecchi, M., "Use of couple-stress theory in elasto-plasticity". Comput. Methods Appl. Mech. Engrg. 136, 205-224 (1996).

7. Ottosen, N. S., Ristinmaa, M. and Ljung, C., "Rayleigh waves obtained by the indeterminate couple-stress theory". European Journal of Mechanics - A/Solids, 19, 929-947 (2000).

8. Fleck, N.A. and Hutchinson, J.W., "Strain gradient plasticity". In J.W. Hutchinson and T.Y. Wu (eds.) Advances in Applied Mechanics, Vol. 33. Academic Press, New York, pp. 295-361 (1997).

9. Fleck, N.A. and Hutchinson, J.W., "A reformulation of strain gradient plasticity". Journal of the Mechanics and Physics of Solids, 49, 2245-2271 (2001).

10. Koiter, W.T., "Couple-stresses in the theory of elasticity, I and II". Proc. Ned. Akad. Wet. (B), 67, 17-44 (1964).

11. Xia, Z.C. and Hutchinson, J.W., "Crack-tip fields in strain gradient plasticity". Journal of the Mechanics and Physics of Solids, 44, 1621-1648 (1996).

12. Huang, Y., Zhang, L., Guo, T. F. and Hwang, K.C., "Mixed Mode near-tip fields for crack in materials with strain-gradient effects". Journal of the Mechanics and Physics of Solids, 45, 439-465 (1997).

13. Huang, Y., Chen, J.Y., Guo, T.F., Zhang, L. and Huang, K.C., "Analytic and numerical studies on Mode I and Mode II fracture in elastic-plastic materials with strain gradient effects". International Journal of Fracture, 100, 1-27 (1999).

14. Gao, H., Huang, Y., Nix, W.D. and Hutchinson, J.W., "Mechanism-based strain gradient plasticity-I. Theory". Journal of the Mechanics and Physics of Solids, 47, 1239-1263 (1999).

15. Chen, J.Y., Wei, Y., Huang, Y., Hutchinson, J.W. and Hwang, K.C., "The crack-tip fields in strain gradient plasticity: the asymptotic and numerical analyses". Engineering Fracture Mechanics, 64, 625-648 (1999).

16. Shi, M.X., Huang, Y., Gao, H. and Hwang, K.C., "Non-existence of separable crack-tip field in mechanism-based strain gradient plasticity". International Journal of Solids and Structures, 37, 5995-6010 (2000).

17. Radi, E., "Strain-gradient effects on steady-state crack growth in linear hardening materials". Journal of the Mechanics and Physics of Solids, 51, 543-573 (2003).

18. Wei, Y. and Hutchinson, J.W., "Steady-state crack growth and work of fracture for solids characterized by strain gradient plasticity". Journal of the Mechanics and Physics of Solids, 45, 1253-1273 (1997).

19. Amazigo J. and Hutchinson J.W., "Crack-tip fields in steady crack-growth with linear strain hardening". Journal of the Mechanics and Physics of Solids, 25, 81-97 (1977).

20. Ponte Castañeda, P., "Asymptotic fields in steady crack growth with linear strain-hardening". Journal of the Mechanics and Physics of Solids, 35, 227-268 (1987).

21. Bigoni, D. and Radi, E., "Asymptotic solution for Mode III crack growth in $\mathrm{J}_{2}$-elastoplasticity with mixed isotropic-kinematic strain hardening". International Journal of Fracture, 77, 77-93 (1996).

22 Vardoulakis, I., Exadaktylos, G. and Aifantis, E., "Gradient elasticity with surface energy: Mode-III crack problem". International Journal of Solids and Structures, 33, 4531-4559 (1996).

23. Zhang, L., Huang, Y., Chen, J.Y. and Hwang, K.C., "The Mode III full-field solution in elastic materials with strain gradient effects". International Journal of Fracture, 92, 325-348 (1998).

24. Unger, D.J. and Aifantis, E.C., "Strain gradient elasticity theory for antiplane shear cracks. Part I: Oscillatory displacements" Theoretical and Applied Fracture Mechanics, 34, 243-252 (2000).

25. Fannjiang, A.C., Chan, Y.-S. and Paulino, G.H., "Strain gradient elasticity for antiplane shear cracks: a hypersingular integrodifferential equation approach". SIAM Journal of Applied Mathematics, 62, 1066-1091 (2002).

26. Radi, E. and Gei, M, "Mode III crack growth in linear hardening materials with strain-gradient effects". International Journal of Fracture, 130, 765-785 (2004). 